\title{
Announcement: Changes to the sections of Physical Review A
}

We are reorganizing and modernizing the sectioning scheme of Physical Review A (PRA). As of July 1, 2020 , the journal will include eight sections instead of the previous seven. The main changes are the split of the Quantum Information section into two sections, the split of the Quantum optics, physics of lasers, nonlinear optics, classical optics section into two, and the consolidation of the Atomic and molecular structure and dynamics; high-precision measurements section and the Atomic and molecular collisions and interactions section into one. Apart from this, there are several other minor rearrangements.

Over time, different subfields within the scope of a journal naturally evolve in different ways as the community evolves; some areas grow while others may shrink. Both the quantum information section and the optics section of the journal have seen very strong growth, and they are now by far the largest sections. Conversely, papers in the areas of atomic structure and atomic collisions have slowly declined in number though not in quality. The editors, therefore, felt it appropriate to acknowledge this fact by updating the journal's sectioning scheme, in order to better reflect the evolving interests of our authors and readers, and to align more closely with the different communities we serve.

The details of the new scheme, as well as a listing of specific topics for each one of the sections, can be found at https://journals.aps.org/pra/authors/guidelines-section-selection-physical-review-a. As stated above, this change in sectioning is meant to be a reorganization and modernization of the way we categorize the manuscripts we publish. It does not imply a change of the scope of the journal. Specifically, we continue to welcome the full breadth of work which is of interest to the AMO and quantum information communities, subject, of course, to the usual quality and other acceptance criteria of the Physical Review.

Published 1 July 2020

DOI: 10.1103/PhysRevA.102.010001 